\title{
Zertifizierung von Darmkrebszentren - eine kritische Standortbestimmung anhand ungeklärter Aspekte
}

\author{
Certification of Colorectal Cancer Units - \\ A Critical Overview on the Basis of Unsettled Aspects
}

Autor

Institut

\section{H. J.C. Klaue}

Klinikum Fichtelgebirge gGmbH, Haus Marktredwitz, Chirurgische Klinik, Allgemein- und Viszeralchirurgie, Marktredwitz, Deutschland

\author{
Schlüsselwörter \\ - kolorektales Karzinom \\ - Ergebnisqualität \\ D Zertifizierung \\ - kolorektale Krebszentren \\ Key words \\ - colorectal carcinoma \\ $\checkmark$ outcome quality/ \\ quality of results \\ - (board) certification \\ - special colorectal cancer \\ units
}

\section{Zusammenfassung \\ $\nabla$}

In der vorliegenden Arbeit wird die Zertifizierung von Darmkrebszentren anhand bisheriger Publikationen und weiterer, wissenschaftlich nicht geklärter Aspekte, welche die Versorgungsqualität beeinflussen, kritisch hinterfragt. Die Darstellung des Themas kann nicht als vollständige und abschließende Bewertung verstanden werden, sondern soll auch anhand bisher wenig beachteter Gesichtspunkte dem Leser Denkanstöße für eine eigene kritische Würdigung des Themas geben. Aufgrund vieler Limitationen der bisher publizierten Literatur und den in der Arbeit dargestellten unklaren Aspekten muss der Sinn der Zertifizierung von Darmkrebszentren angezweifelt werden.

Fünf Jahre nach der Zertifizierung des ersten Darmkrebszentrums in Deutschland ist es an der Zeit, den Sinn dieser Zertifizierungen kritisch zu hinterfragen. Zu diesem Zweck sollen zu dieser Thematik bisher publizierte Arbeiten ebenso wie weitere bislang kaum beachtete Faktoren, die einen Einfluss auf die Qualität der Behandlung onkologischer Patienten haben könnten, diskutiert werden. Die Komplexität der Problematik einer bestmöglichen Versorgung unserer Patienten soll damit ansatzweise aufgezeigt werden. Sinnvoll ist hier zunächst, die von den Befürwortern der Zertifizierung und Zentrenbildung postulierten Ziele in Erinnerung zu rufen. Eine umfassende Übersicht zu diesem Thema findet sich, unter Federführung der Deutschen Krebsgesellschaft e.V. Berlin publiziert, im Februarheft des Onkologen aus diesem Jahr [1]. Zentrales Anliegen ist die Verbesserung der Behandlungsqualität Krebskranker in Deutschland. Die grundlegende Notwendigkeit der Einführung von Darmkrebszentren wird unter anderem mit der CONCORDStudie [2] begründet, aus der eine im internationalen Vergleich nur mittelmäßige Behandlung

\section{Abstract \\ $\nabla$}

In this contribution the rational of colorectal cancer centre certification is discussed on the basis of previously published literature and several unsettled factors which are not yet considered as influencing the quality of cancer treatment. The representation of the theme cannot be comprehensive or complete. The readers should be stimulated by one or the other topic to reach their own critical assessment. Due to several limitations of the published literature and the various unsettled aspects, the purpose of colorectal cancer centre certification is called into question.

onkologischer Patienten in Deutschland abgeleitet wird. Weiterhin führen die Autoren aus, dass in absehbarer Zeit alle Krebspatienten nur noch in entsprechend zertifizierten Zentren behandelt werden sollten, um optimale Behandlungsergebnisse zu gewährleisten. So wird behauptet, dass die Prognose von Tumorpatienten sehr von der Behandlung durch ihre Ärzte abhängig sei. Der Bürger, zum Patienten geworden, müsse vor inadäquater Behandlung geschützt werden: Vor Therapeuten, die den zu erwartenden Anforderungen an die Behandlungsqualität nicht gerecht würden. Diese seien jedoch nur zu identifizieren und zukünftig auszuschließen, wenn das Schicksal der von ihnen behandelten Patienten zu ihnen zurückzuverfolgen sei [1]. Insofern sei die Dokumentation und Offenlegung von Behandlungsparametern notwendig. Es wird weiterhin behauptet, dass nur eine Zertifizierung sicherstellen könne, dass den Vorgaben von Leitlinien und den strukturellen Anforderungen sowie der Ergebnisqualität Rechnung getragen würde. Konsequenterweise wird gefordert, dass von den derzeitigen Kriterien der Zertifizierung in Zukunft keine 
Abstriche gemacht werden dürfen, wenn die Behandlungsdaten im Quervergleich für Rezertifizierungen herangezogen werden. Dies wird in Zukunft zu einer gewissen Zentralisierung führen, vor allem aber zum Ausschluss derjenigen Kliniken, die sich nicht einer Zertifizierung unterwerfen [1]. Nicht zuletzt wird in dieser Arbeit auch das Thema der Mindestmengen sowie der „High Volume Hospitals" angesprochen, welches der Zentrenbildung gesundheitspolitisch zugrunde liegt, und gefordert, Mindestmengen zu definieren, da zumindest die Tatsache belegt zu sein scheint, dass „High-Volume-Kliniken“ mit Komplikationen besser umgehen können [1].

\section{Welche Anforderungen stellen Gesetzgebung und Rechtsprechung? \\ $\nabla$}

Die ethisch-moralischen Grundsätze unseres ärztlichen Handelns fordern selbstverständlich die optimale Behandlung eines jeden erkrankten Patienten. Nun bedeutet optimal das unter gegebenen Bedingungen höchst erreichbare Ergebnis: Die Diskussion, ob eine gewissermaßen optimale Behandlung in unserem Lande unter den bei uns gegebenen Bedingungen nicht ohnehin vorliegt und wir nicht vielmehr an eine Optimierung hin zu einer idealen Versorgung unserer Patienten denken, würde hier aber viel zu weit führen. Beschränken wir uns auf die legislativen und judikativen Vorgaben, so definiert das SGBV in $\S 12$, dass die zu erbringenden Leistungen ausreichend, zweckmäßig und wirtschaftlich sein müssen; sie dürfen das Maß des Notwendigen nicht überschreiten.

Der Bundesgerichtshof fordert für die Behandlung von Patienten lediglich das Maß an Kenntnis und Können eines durchschnittlichen, erfahrenen Facharztes [3].

Eine optimale Behandlung von Patienten wird also weder von der Gesetzgebung noch von der Rechtssprechung eingefordert.

Die unsererseits ethisch-moralisch begründete Forderung nach einer stets optimalen Therapie aller Patienten erreicht hingegen ihre erste Limitierung schon in der Bereitstellung der dafür dann prinzipiell notwendigen, optimalen Finanzierung.

Zunächst ist die Frage zu diskutieren, ob eine Krebszentrenbildung in Deutschland überhaupt notwendig ist. Die Befürworter einer Zertifizierung von Darmkrebszentren [1] begründen die Notwendigkeit unter anderem mit der CONCORD-Studie [2] und interpretieren diese Studie so, dass die Behandlungsqualität in Deutschland im internationalen Vergleich nur im Mittelmaß liege. Anzumerken sind hierzu erstens, dass in der CONCORD-Studie keine Daten für Deutschland zur Verfügung standen, sondern lediglich das Krebsregister des Saarlandes ausgewertet wurde. Ob die im Saarland erreichten Ergebnisse aber repräsentativ für ganz Deutschland sind, ist unbekannt und kann bezweifelt werden. Und zweitens, dass in der CONCORD-Studie lediglich Daten von 101 Krebsregistern aus 31 Ländern ausgewertet wurden. Ganz Afrika war nur durch ein einziges Register aus einem algerischen Departement vertreten, Südamerika und Asien nur durch eines aus Kuba, zwei brasilianische und drei japanische. Das mit Abstand schlechteste Überleben der Krebspatienten wurde jeweils in Algerien erreicht. Es ist anzunehmen, dass Deutschland bei einer Auswertung von allen 193 Ländern weltweit sicher nicht im Mittelfeld, sondern in der Spitzengruppe zu finden wäre. Im Vergleich mit ausschließlich Industrienationen liegen die Ergebnisse des Saarlandes allerdings in der Tat nur im Mittelfeld der ausgewerteten Nationen (beim kolorektalen Karzinom wurde bei weiblichen Patienten der 12., bei männlichen der 14. Platz erreicht) [2].

\section{Stellt nur ein Zertifikat eine qualitativ hochwertige Versorgung sicher?}

$\nabla$

Der anfangs zitierten These, nur eine Zertifizierung könne sicherstellen, dass den Vorgaben von Leitlinien und der Ergebnisqualität in der Versorgung Krebskranker Rechnung getragen werde, kann so nicht zugestimmt werden. Zunächst basiert die Ansicht, dass Patienten mit kolorektalen Karzinomen an Zentren besser behandelt würden, auf der Annahme der Gültigkeit des „High Volume Hospital“ und „High Volume Surgeon Caseload“ Effekts. Wie in einer Übersichtsarbeit [4] nach ausführlicher Diskussion der Literatur festgestellt wurde, rechtfertigen die vorliegenden Daten aber insbesondere für das kolorektale Karzinom nicht, Mindestvorgaben oder -mengen festzuschreiben. Problematisch ist die Interpretation der vorliegenden Daten insbesondere aufgrund der Heterogenität der Definition von „Low-Volume“- und „High-Volume“-Kliniken respektive Chirurgen, der überwiegend fehlenden Risikostratifizierung im untersuchten Patientengut, der retrospektiven Auswertung von weitgehend administrativen Datensammlungen, der unterschiedlichen Einweisungspraxis (elektive vs. Notfallpatienten) und nicht zuletzt der fehlenden Berücksichtigung weiterer Faktoren wie interdisziplinärer $\mathrm{Zu}$ sammenarbeit, Tumorboards und der postoperativen intensivmedizinischen Nachbehandlungsmöglichkeiten [4-7]. Die Problematik der Interpretation der vorliegenden Literatur, insbesondere für die kolorektale Chirurgie, wurde auch von Weber und Link ausführlich diskutiert [6] und festgehalten, in der Kolonund Rektumchirurgie sei der Unterschied im Outcome zwischen „Low-Volume“- und „High-Volume“-Zentren deutlich geringer als in der Pankreas- und Ösophaguschirurgie und es seien mehr prospektiv kontrollierte Studien notwendig, um den Einfluss der verschiedenen Faktoren genauer zu definieren.

Wiederholt wurde publiziert, Kliniken mit vergleichsweise geringen Fallzahlen könnten eine ebenso gute Ergebnisqualität wie sogenannte „High-Volume“-Zentren erzielen [7]. Auch nicht speziell als Darmkrebszentrum zertifizierte Kliniken können eine ordentliche Ergebnisqualität abliefern, wie die $\bigcirc$ Tab. 1 mit den Daten der eigenen Klinik demonstriert.

Weiterhin wirft der Vergleich der chirurgischen Ergebnisqualität zertifizierter deutscher Darmkrebszentren mit den Ergebnisdaten der multizentrischen Beobachtungsstudie des An-Instituts für Qualitätssicherung in der operativen Medizin die Frage auf, ob eine Zertifizierung wirklich notwendig ist, eine adäquate Ergebnisqualität zu erreichen, mithin durch die Zertifizierung überhaupt ein positiver Effekt für die flächendeckende Versorgung der Patienten erreicht wird [8, 9]. So zeigen die Daten des BenchmarkBerichtes der Onkozert Darmkrebszentren für das Jahr 2010, dass von 22 Qualitätsmerkmalen bei der Kennzahlauswertung bei 21 Qualitätsparametern die Sollvorgabe von den zertifizierten Kliniken in einem Range von 9,9-72,7\% nicht erfüllt wurde! Bei 17844 primären kolorektalen Karzinomen lag die Anastomoseninsuffizienzrate nach elektiven Eingriffen am Kolon im Mittel bei $4,9 \%$ und damit deutlich über der geforderten Sollvorgabe von $\leq 3 \%$ [8]. Das flächendeckend eine Anastomoseninsuffizienzrate von 3\% bei Kolonresektionen wegen eines Karzinoms in Deutschland aber durchaus erreichbar ist, zeigen die Daten des An-Instituts an 28271 Patienten mit Kolonkarzinomen [9]. Auch wenn der einfache univariate Vergleich dieser beiden Kollektive streng genommen nicht durchgeführt werden darf, ist aufgrund der großen Fallzahl in beiden Kollektiven davon auszugehen, dass die Behandlung von Patienten in nicht zertifizierten Kliniken jedenfalls nicht schlechter ist als in zertifizierten Zentren. 
Tab.1 Ergebnisse der kolorektalen Chirurgie, Klinikum Fichtelgebirge, Abteilung Allgemein- und Viszeralchirurgie (1.3.2007-18.5.2011).

\begin{tabular}{|c|c|}
\hline Mortalität elektiver Operationen ( $n=437)$ & $2,9 \%$ \\
\hline Mortalität frühelektiver Operationen ( $n=144)$ & $4,1 \%$ \\
\hline Mortalität Notfalloperationen $(n=179)$ & $15,0 \%$ \\
\hline Gesamtmortalität $(n=760)$ & $6,0 \%$ \\
\hline $\begin{array}{l}\text { Anastomoseninsuffizienzen (19/674): } \\
\text { Gesamtinsuffizienzrate }\end{array}$ & $2,8 \%$ \\
\hline $\begin{array}{l}\text { Kolonkarzinom - Anastomoseninsuffizienzen } \\
(4 / 234)\end{array}$ & $1,7 \%$ \\
\hline $\begin{array}{l}\text { Divertikulitisresektionen - Anastomosen- } \\
\text { insuffizienzen }(5 / 184)\end{array}$ & $2,7 \%$ \\
\hline andere Kolonresektionen (4/ 169) & $2,3 \%$ \\
\hline $\begin{array}{l}\text { Rektumkarzinomresektionen: } \\
\text { Gesamtinsuffizienzrate }(6 / 87) \\
\text { anteriore Resektionen (ohne protektives }\end{array}$ & $6,9 \%$ \\
\hline $\begin{array}{l}\text { Stoma) }(2 / 33) \\
\text { tiefe anteriore Resektionen (ohne protektives }\end{array}$ & $6,1 \%$ \\
\hline $\begin{array}{l}\text { Stoma) }(1 / 28) \\
\text { tiefe anteriore Resektionen (mit protektivem } \\
\text { doppelläufigem lleostoma }(3 / 26)\end{array}$ & $11,5 \%$ \\
\hline Rektumkarzinome insgesamt $(n=119)$ & \\
\hline davon: Rektumamputation nach Miles & $17,6 \%$ \\
\hline Hartmann-Operationen & $12,6 \%$ \\
\hline Resektion mit Anastomosen & $69,8 \%$ \\
\hline MERCURY GradI & $95,7 \%$ \\
\hline kolorektales Karzinom, R0-Resektionsrate: & $\begin{array}{l}94,3 \% \text { von } \\
\text { ( } n=366 \text { Patienten) }\end{array}$ \\
\hline Lymphknotenresektionsrate, durchschnittlich: & $\begin{array}{l}23,9 / \text { Fall } \\
\text { Range yp } 10-p 65 \\
\text { in } 99,4 \%>12\end{array}$ \\
\hline $\begin{array}{l}\text { adjuvante Chemotherapie im UICC-III-Stadium, } \\
\text { Häufigkeit: }\end{array}$ & $72 \%$ \\
\hline
\end{tabular}

Dies führt zur Frage, ob das primäre Ziel jeder Zentrenbildung, nämlich die qualitative Verbesserung der Patientenversorgung, nur per Zertifizierung bzw. überhaupt durch Zertifizierung von Zentren erreicht werden kann. In der Literatur wird immer wieder darauf hingewiesen, dass die multidisziplinäre Versorgung onkologischer Patienten entscheidend für das Behandlungsergebnis ist $[1,4,6]$. Dies bedeutet jedoch nicht, zertifizierte Zentren führen per se eine bessere interdisziplinäre Versorgung als nicht zertifizierte Kliniken durch. Qualitätsmängel entstehen offensichtlich durch Missachtung der evidenzbasierten medizinischen Leitlinien $[4,10]$, deren Befolgung aber keine Zertifizierung voraussetzt. Wiederum zeigen die vom An-Institut erhobenen Daten, dass die Versorgungsqualität in Deutschland im zeitlichen Verlauf durch bessere Beachtung der Leitlinienvorgaben auch ohne Zertifizierungen gesteigert wurde [10].

\section{Welche Faktoren beeinflussen die Leistungsfähigkeit eines Chirurgen? \\ $\nabla$}

Die Kenntnis und Leistungsfähigkeit eines (chirurgischen) Therapeuten werden durch vielfältige Faktoren bestimmt, von denen etliche in der Literatur zum Teil untersucht und beschrieben, andere jedoch bisher noch nicht ausreichend beachtet wurden (D Tab.2).

Beispielhaft für anerkannte Faktoren ist hier die Bildung von spezialisierten „Colorectal Cancer Units“ kurz zu diskutieren,
Tab.2 Faktoren, welche die Ergebnisqualität eines Chirurgen mitbestimmen.

\begin{tabular}{ll}
$\begin{array}{l}\text { A } \\
\text { in Studien untersuchte }\end{array}$ & $\begin{array}{l}\text { B } \\
\text { in Studien noch nicht } \\
\text { untersuchte }\end{array}$ \\
Training und Teaching & individuelle Begabung \\
\hline Supervision & Arbeitsklima, beruflicher Stress \\
\hline chirurgische Spezialisierung & persönliche Probleme \\
\hline Fallzahl des Chirurgen & Erfolgsdruck \\
\hline Fallzahl des Klinikums & $\begin{array}{l}\text { Motivation, persönliche } \\
\text { Einstellung }\end{array}$
\end{tabular}

die von einigen Autoren als letzter logischer Schritt nach erfolgter Etablierung von speziellem Training, der Überwachung durch externe Experten, der Ausbildung an Exzellenzzentren, der Weiterbildung in Viszeralchirurgie und der möglichen Schaffung einer Subspezialität „Kolorektale Chirurgie“ angesehen werden [11].

Insgesamt 3 Artikel wurden zum Thema Etablierung von speziellen „Colorectal Cancer Units“ publiziert und werden in der Literatur zitiert, um die bessere Versorgung der Patienten durch solche Einrichtungen beispielhaft zu belegen [11]. Alle 3 Publikationen demonstrierten eine Verbesserung des chirurgischen Ergebnisses, in dem Patienten durch eine kleinere Anzahl von speziell trainierten Chirurgen versorgt wurden [12-14]. Hierzu muss aber kritisch angemerkt werden, dass in allen Arbeiten Patientengruppen verglichen wurden, die vor Einführung der total mesorektalen Exzision (TME) an den Kliniken ohne TME und, nach Training der publizierenden Chirurgen, mit TME an den neu geschaffenen Zentren versorgt wurden. Diese Arbeiten beweisen also die Verbesserung der Patientenversorgung durch die Einführung der TME, nicht jedoch, dass die Gründung spezialisierter „Colorectal Cancer Units“ die Prognose der Patienten zusätzlich verbessert. Übereinstimmend zeigen sie die Reduktion der Rektumamputationsrate nach Miles und die der Lokalrezidivrate nach Rektumoperationen in einem Umfang, der auch schon von Lehander Martling et al. als reiner Effekt eines chirurgischen Trainingprogramms im Stockholm-Trial publiziert wurde [15]. Ein Vorteil von „Colorectal Cancer Units“ kann nur durch den Vergleich von TME-Operationsergebnissen von Kliniken mit und ohne spezialisierte „Units“ nachgewiesen werden.

Bei kritischer Durchsicht der Literatur stellt sich somit die Frage, ob nicht der Schlüssel zur verbesserten Versorgung von Patienten mit Rektumkarzinomen allein in der Teilnahme an verpflichtenden Fortbildungen zur Etablierung der TME [15], also der operativen Fortbildung, liegt bzw. lag.

In ganz anderer Richtung beachtenswert ist aber die Aussage der spanischen Kollegen, die in ihrer Publikation mitteilen, dass die 4 Chirurgen der Unit of Coloproctology seit 1992 ausschließlich kolorektale Chirurgie betreiben, also bereit waren, ihre anderen Patienten an diejenigen Kollegen abzutreten, die auf die kolorektale Chirurgie verzichteten [14]. Diese radikal erscheinende Konsequenz ist aber auch folgerichtig, wenn man an die Gültigkeit der Fallzahl-Effekte glaubt. Sie sollte aber auch hinsichtlich ihrer weiteren Auswirkungen, z. B. auf die Notfallversorgung, betrachtet und kritisch hinterfragt werden. Potenzielle Vorteile einer Zentrumsversorgung müssten im Gesamtkontext der allgemeinen Patientenversorgung evaluiert und dokumentiert werden, um Nachteile an anderer Stelle sicher auszuschließen; ein fast unmöglich erscheinendes Unterfangen. 


\section{Beeinflusst die Offenlegung von Behandlungs- ergebnissen die Qualität der Therapie? \\ $\nabla$}

Als Beispiel für einen bisher noch nicht publizierten Parameter, der die Ergebnisse von Chirurgen mitbestimmt, soll an dieser Stelle die Auswirkung des Erfolgsdruckes, der durch Offenlegung von Qualitätsparametern entsteht, demonstriert werden:

Die $\triangle$ Tab. 3 zeigt die anonymisierten Daten einer klinikinternen, operateursbezogenen Komplikationsstatistik aus dem Bereich der kolorektalen Chirurgie, die wir am Diakonie-Krankenhaus Rotenburg/Wümme zwischen 1998 und 2002 erhoben hatten. Aufgrund einer im Sommer 1998 aufgefallenen, ungewöhnlich hohen Rate an Anastomoseninsuffizienzen wurden die Zirkulärstapler der Firma I (nachfolgend wurde von der Firma ein Produktionsfehler bei einer Charge angegeben) im September 1998 auf Zirkulärstapler der Firma II gewechselt. Die Insuffizienzrate der Resektionen am linken Kolon und Rektum fiel in den nächsten Monaten von $16,6 \%$ auf $8 \%$ ab.

Ab September 1998 wurde eine operateursbezogene, prospektive Datenerhebung durchgeführt. Im Laufe der Jahre zeigte sich bei recht stabiler Insuffizienzrate eine Zunahme der Diskontinuitätsresektionen nach Hartmann von gut 11\% im Jahre 1999 auf $19 \%$ im Jahre 2001. Daraufhin wurde die operateursbezogene Auswertung unterlassen und für das Jahr 2002 lediglich die Gesamtklinik-Insuffizienzrate und die Rate an Hartmann'schen Operationen bestimmt, wobei sich zeigte, dass sowohl die Insuffizienzrate auf 2,9\% als auch die Rate an Hartmann'schen Operationen auf 8,6\% abfiel. Auch wenn dies nicht risikoadjustierte, rein deskriptive Daten sind, muss diskutiert werden, ob die durch die Offenlegung von operateursbezogenen Komplikationsdaten entstehenden psychischen Effekte die Operateure belastet haben und zur Vermeidung einer möglichen Insuffizienz deshalb eher auf eine Anus-praeter-Anlage zurückgegriffen wurde. Der schlagartige Rückgang an Hartmann'schen Operationen zwischen 2001 und 2002 um mehr als 50\% spricht für einen gewissen „befreienden Effekt", der sich erstaunlicherweise auch in einer extrem niedrigen Insuffizienzrate bei den Patienten widerspiegelte.

Auch wenn diese Daten nicht als Beweis für die Auswirkungen eines Erfolgdruckes auf die operativen Ergebnisse von Chirurgen gewertet werden können, so ist ein gewisser Effekt doch nicht völlig von der Hand zu weisen. Ähnlich könnten sich theoretisch auch Faktoren wie das Arbeitsklima an einer Klinik oder privater Stress auswirken. Niemand wird bestreiten, dass die individuelle Begabung von Chirurgen unterschiedlich ist und ebenso wie die

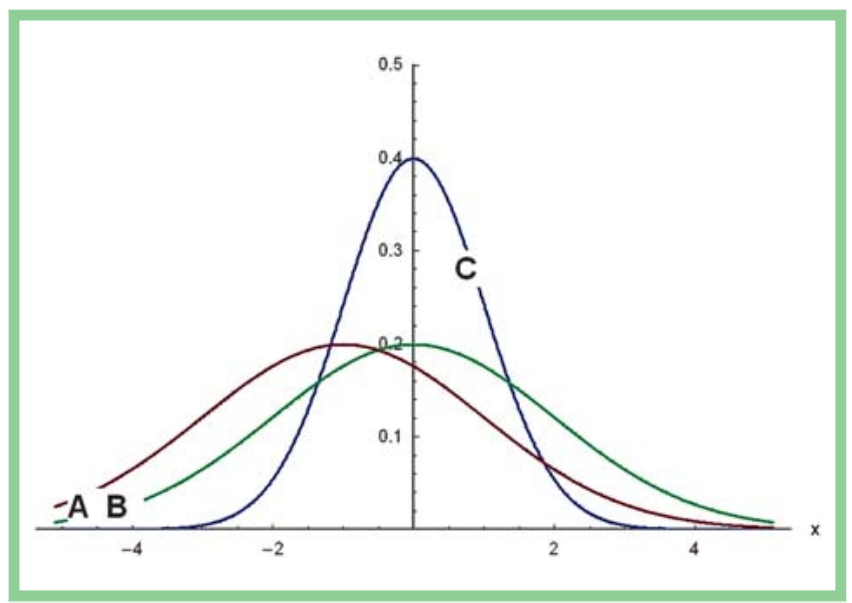

Abb.1 Hypothetische Verteilung der Ergebnisqualität von Chirurgen.

persönliche Motivation Einfluss auf die Arbeitsergebnisse nimmt. Insgesamt kann man postulieren, dass die in $\oslash$ Tab. 2 aufgeführten Faktoren zu einer wie auch immer verlaufenden Gauß'schen Verteilungskurve der Leistungsfähigkeit von chirurgischen Therapeuten führen ( $\boldsymbol{Q} \mathbf{A b b} \mathbf{b} \mathbf{1})$. Betrachtet man die Ergebnisse des Benchmarking-Berichtes von Onkozert für das Jahr 2010, so wird man für viele Qualitätsparameter eine relativ breit verlaufende Verteilungskurve erhalten (ähnlich der Kurven A und B). Es ist zu beachten, dass individuelles Können wie Kenntnis im Spitzenbereich sicher nur von wenigen Chirurgen erreicht werden kann.

Die Einführung neuer, besserer Therapiekonzepte (z. B. TME) und deren flächendeckende Verbreitung führt entsprechend den individuellen Möglichkeiten der Chirurgen zu einer relevanten Verbesserung der Patientenversorgung, die Ergebniskurve A würde zur Kurve B verschoben werden.

Erstaunlicherweise finden sich überhaupt keine Publikationen über einen „High-Volume“-Effekt für Onkologen oder Strahlentherapeuten. Mutmaßlich differieren aber auch deren Behandlungsergebnisse, schon da die Diagnose und Therapie von teils fatalen Komplikationen sicher nicht jedem Therapeuten gleich gut gelingt. Es stellt sich die Frage, was mit Zentren geschehen soll, an denen z. B. „gute“ Chirurgen und Onkologen (jeweils Kurve C) mit z. B. „schlechten“ Strahlentherapeuten (Kurve A statt B) zusammenarbeiten.

Tab.3 Operateursbezogene Komplikationsstatistik kolorektaler Anastomoseninsuffizienzen der Klinik für Allgemein-, Viszeral- und Thoraxchirurgie des DiakonieKrankenhauses Rotenburg/Wümme (1.2.1998 bis 31.12.2002).

\begin{tabular}{|c|c|c|c|c|c|c|c|}
\hline \multirow{2}{*}{$\begin{array}{l}\text { Operateur } \\
\text { Jahr }\end{array}$} & \multirow{2}{*}{$\begin{array}{l}\text { Anzahl OP mit } \\
\text { Anastomose } \\
\text { 1998-2001 }\end{array}$} & \multicolumn{6}{|c|}{ Anastomoseninsuffizienzrate (\%) } \\
\hline & & \multicolumn{5}{|l|}{ 1998-2001 } & 2002 \\
\hline A & 79 & 2,5 & 6,6 & 0,0 & 0,0 & 4,7 & 1 \\
\hline B & 127 & 7,8 & 13,0 & 5,1 & 10,0 & 6,6 & 1 \\
\hline C & 192 & 7,2 & 7,1 & 0,0 & 7,8 & 12,9 & 1 \\
\hline D & 74 & 12,1 & 14,2 & 10,5 & 20,0 & 11,5 & 1 \\
\hline $\mathrm{E}$ & 124 & 6,4 & 10,0 & 11,4 & 0,0 & 0,0 & 1 \\
\hline $\mathrm{F}$ & 13 & 0,0 & 1 & 1 & 1 & 0,0 & 1 \\
\hline Summe Klinik & 609 & 7,0 & 10,3 & 4,9 & 7,5 & 7,7 & 2,9 \\
\hline \multicolumn{8}{|c|}{ Hartmann-OP (\%) } \\
\hline Summe Klinik & 1 & 1 & 1 & 11,2 & 17,6 & 19,3 & 8,6 \\
\hline
\end{tabular}


Die Kernfrage ist hier doch, wie weit man chirurgische Spezialisierungen vorantreiben will und kann, wenn man bedenkt, dass die „Ressource Chirurg“ begrenzt ist und eine qualitativ hochstehende Notdienstversorgung auch flächendeckend aufrecht erhalten werden muss. Außerdem muss ein unterdurchschnittlicher „Kolorektalchirurg“, der fortan keine Darmchirurgie mehr betreiben dürfte, ja nicht zwangsläufig ein guter „Ösophagus“-, „Gallenblasen“- oder „Hernienchirurg“ sein, also in diesen Versorgungsbereichen zum Vorteil der Gesellschaft einsetzbar sein.

Resultierend ist an dieser Stelle kritisch zu hinterfragen, wer denn nach strikter Anwendung des vom BGH geforderten Kriteriums eines durchschnittlichen erfahrenen Facharztes diejenigen Patienten in Zukunft versorgen soll, die von den 50\% unterdurchschnittlichen Chirurgen bisher versorgt wurden, wenn diese von der weiteren Versorgung ausgeschlossen würden. Welcher Spezialist verfügt über entsprechend freie Valenzen seiner Arbeitskapazität, um die doppelte Anzahl von Patienten zu behandeln? Die Versorgungsrealität ist in diesem Kontext zwingend zu beachten.

Im übrigen erfordert der Ausschluss von Chirurgen natürlich eine unabhängige, stringente, risikoadjustierte Datenerhebung bezüglich einer ganzen Reihe von Qualitätsparametern für jeden einzelnen Chirurgen bzw. eine einzelne Klinik.

\section{Wird Benchmarking effektive Konsequenzen ermöglichen? \\ $\nabla$}

Dies führt wiederum unweigerlich zur Frage, ob risikoadjustierte Daten für einzelne Operateure oder Kliniken in einem signifikanten Umfang, also in justiziablem Sinne beweisende Daten überhaupt erhoben werden können, sodass wirksame Konsequenzen gezogen werden könnten. Eine ausführliche Diskussion zur Problematik der bisher unmöglichen Festsetzung von Mindestfallzahlen im Rahmen der Versorgung von kolorektalen Karzinomen findet sich bei Weber u. Link [6]. Setzt man einmal die von Onkozert geforderten 50 Resektionen jährlich bei kolorektalen Karzinomen pro Klinik zugrunde, würde man ca. 10 Jahre benötigen, um eine statistisch ausreichende Grunddatenmenge bezüglich der Anastomoseninsuffizienz- und Reinterventionsrate einer Klinik zu erlangen. Um das 5-Jahres-Überleben dieser Patientengruppe sicher zu definieren, ist ein Zeitraum von 15 Jahren zur Datenerhebung zu veranschlagen. Berücksichtigt man, dass ggf. erst nach dieser Zeit Rückmeldungen zu Verbesserungsvorschlägen gemacht werden können und der Klinik Gelegenheit zur Verbesserung inklusive erneuter Datenerhebung zu gewähren ist, würden nochmals mindestens drei bis sechs Jahre vergehen, bis ein letztendlich justiziabler Datensatz erhoben worden ist. Das Berufsleben des verantwortlichen Chefarztes ist zu diesem Zeitpunkt in aller Regel beendet, sodass ein Effekt für die Versorgung der Patienten so nicht zu erreichen ist.

Zur Begründung des Sinnes und des Erfolges der Zertifizierungen von Darmkrebszentren verweisen die Befürworter unter Anderem auch auf die häufigere Durchführung einer adjuvanten Chemotherapie im UICC-Stadium III, da an zertifizierten Darmkrebszentren im Mittel 73,8\% der Patienten, bundesweit aber nur $61,2 \%$ der Patienten die indizierte adjuvante Chemotherapie tatsächlich erhalten $[1,8]$. Ob bei diesen rein deskriptiven Daten ein statistisch signifikanter Unterschied besteht, kann nicht beurteilt werden. Bei effektiver Verbesserung des 5-Jahres-Überlebens von ca. 10 bis $15 \%$ durch eine adjuvante Chemotherapie (FOGT-1, CCA-0,1, MOSAIC-Studien) hätten bei 12,6\% mehr behandelten
Patienten nur ca. 1,8\% der Patienten des UICC-Stadium III eine bessere Prognose. Bei einem Anteil des UICC-Stadium III von ca. $30 \%$ am gesamten Patientengut würden nicht einmal $1 \%$ aller Patienten mit kolorektalen Karzinomen von einer Zentrumsbehandlung profitieren. Hierbei ist zu berücksichtigen, dass Patienten trotz Empfehlung der behandelnden Ärzte nicht immer eine leitliniengerechte Therapie durchführen lassen. So haben an der eigenen Klinik 21\% derjenigen Patienten, denen eine adjuvante Chemotherapie im UICC-Stadium III empfohlen wurde (und für die ein Termin beim behandelnden Onkologen schon vor Entlassung vereinbart war), diese Therapie nicht durchführen lassen. Sieben Prozent der eigenen Patienten waren aufgrund von Komorbiditäten oder einem deutlich reduzierten Allgemeinzustand für eine adjuvante Chemotherapie laut Beschluss der Tumorkonferenz nicht geeignet.

\section{Haben Ärzte und Patienten differente Ansichten über Nutzen und Risiko?}

In diesem Zusammenhang ist ein weiterer Aspekt höchst interessant, der in der Literatur noch nicht ausreichend gewürdigt wurde. So umfasst die leitliniengerechte Behandlung eines Rektumkarzinoms der UICC-Stadien II und III eine neoadjuvante RadioChemotherapie, die auch im zunehmenden Umfang durchgeführt wird [10], und für die Onkozert eine Sollvorgabe von $\geq 80 \%$ für die Darmkrebszentren vorgibt: Bei einer umfassenden Aufklärung über die Vor- und Nachteile dieser Therapie inklusive des erreichbaren Gesamtüberlebens würden sich aber $86 \%$ der Befragten (u.a. 93\% der Laien, 74\% der Mediziner, $91 \%$ der befragten Gesundheitspolitiker) gegen die Durchführung einer neoadjuvanten Radio-Chemotherapie entscheiden [16]. Die Diskrepanz zwischen den tatsächlich applizierten Radio-Chemotherapien und den Ergebnissen der zitierten Studie ist beachtlich. An dieser Stelle ist daran zu erinnern, dass jeder ärztliche Eingriff einer Einwilligung des Patienten bedarf und neben den zu erwartenden Heilungschancen auch die mit dem Eingriff verbundenen Gefahren und Risiken wie auch die Vor- und Nachteile alternativer Behandlungsmethoden in der Aufklärung zu erwähnen sind $[17,18]$. Beachtenswert ist in diesem Zusammenhang auch die Angabe einiger der Befragten, dass Angehörigen von ihnen, die schon an einem Rektumkarzinom erkrankt waren, diese umfassende Information nicht zur Verfügung gestanden hatte und sie sich ggf. für eine andere Behandlung entschieden hätten [16].

\section{Werden die aufgestellten Kriterien der Zertifizierung stringent eingehalten? $\nabla$}

Da Zertifikate ein nicht unwesentliches Marketinginstrument darstellen, ist der Umgang mit Zertifizierungsprozessen kritisch zu überwachen.

Wie oben schon ausgeführt, wollen die Befürworter von Zentrumszertifizierungen durch wiederholte Audits und Überprüfung der Behandlungsqualität zur Verbesserung der Patientenversorgung beitragen. Von den selbst aufgestellten Kriterien der Zertifizierung dürften keine Abstriche gemacht werden, wenn Quervergleiche für die erfolgreiche Rezertifizierung herangezogen werden sollen [1]. Es erstaunt daher, dass im Benchmarking-Bericht für das Jahr 2010 für den Qualitätsindikator Anastomoseninsuffizienzen bei elektiven Kolonresektionen für Audits ab dem Jahr 2011 die Definition der Anastomoseninsuffizienz durch die Beschränkung auf reinterventionspflichtige In- 
suffizienzen präzisiert und darüber hinaus die Sollvorgabe von $\leq 3 \%$ auf $\leq 6 \%$ verändert werden soll [8]. Diese Entscheidung steht den Zielen der Qualitätsverbesserung der Patientenversorgung diametral gegenüber. Hier werden in Zukunft für elektive Kolonresektionen Insuffizienzraten in einer Größenordnung geduldet, die an anderen Kliniken für Rektumresektionen erreicht werden [10]. Da eine Insuffizienzrate von $3 \%$ flächendeckend in Deutschland erreichbar ist [9], ist insbesondere zu kritisieren, dass sich die DKG nicht an ihre eigene Vorgabe, keine Abstriche von den erstellten Kriterien der Zertifikation zu dulden, hält. Folgt man dem Zentrumsgedanken konsequent, so sollte man von den Zentren regelmäßig überdurchschnittlich gute Ergebnisse erwarten können, damit sie ihren Titel rechtfertigen. Die Zielvorgaben sollten entsprechend eigentlich in Richtung $\leq 2 \%$ verändert werden, um gegenüber den flächendeckend erreichten Ergebnissen eine Qualitätssteigerung zu erzielen.

Bei aller Fokussierung auf die operative Ergebnisqualität einzelner Chirurgen oder Kliniken darf nicht vergessen werden, dass die Prognose des kolorektalen Karzinoms im Wesentlichen vom primären TNM-Stadium des Tumors, dem Patientenalter und den Begleiterkrankungen des Patienten abhängig ist [11]. Eine multivariate statistische Untersuchung von mehr als $22400 \mathrm{~Pa}$ tienten ergab einen nur marginalen Einfluss des Caseloads des Chirurgen auf die postoperative Mortalität [19]. In dieser Studie konnte die Wahrscheinlich der postoperativen Mortalität (BasisMortalitätsrate 12 / 1000) durch einen „High-Volume“-Chirurgen um lediglich 1/1000 Fällen und durch ein „High-Volume“-Hospital um knapp 2/1000 Fällen verringert werden. In einer kürzlich publizierten Studie wurde festgestellt, dass bei Überprüfung der operativen Qualität der teilnehmenden Chirurgen mittels vorzulegender Videoaufnahmen von Operationen keine Unterschiede zwischen „Low“-, ,Medium“- oder „High-Volume“-Chirurgen bezüglich der Komplikationsrate und des onkologischen Langzeitergebnisses nachweisbar waren [20].

Dass die Unterteilung von Kliniken in „Low“-, „Medium“- und „High-Volume“-Zentren anhand ihrer Fallzahlen generell problematisch ist, da hierfür keine ausreichende evidenzbasierte wissenschaftliche Basis vorhanden ist, wurde auch schon für die komplexeren Eingriffe der Pankreas- [21] und Magenkarzinomchirurgie [22] diskutiert. Statistisch signifikante FallzahlSchwellenwerte konnten selbst hier ebenso wenig identifiziert werden wie auch keine einheitliche Definition von „Low“-, „Medium“- und „High-Volume“-Kliniken erstellt werden konnte [21, 22].

Dass eine per wiederkehrender Audits überprüfte Zertifizierung Arbeitsabläufe in Kliniken und die Befolgung von medizinischen Leitlinien überprüfen und damit ggf. auch sichern kann, steht außer Frage. Ein realer Vorteil ist aber nur dann gegeben, wenn im Vergleich zu nicht zertifizierten Kliniken ein relevanter, sprich signifikanter Unterschied in der Ergebnisqualität bestehen würde. In Anbetracht des letzten Benchmark-Berichtes [8] muss ein solcher Vorteil infrage gestellt werden.

\section{Welche Nachteile könnten durch Zertifizierungen entstehen? \\ $\nabla$}

Schließlich muss diskutiert werden, ob die Konzentration von medizinischen Leistungen in Zentren noch weitere nachteilige Effekte haben könnte. Theoretisch benötigten wir in Deutschland für unsere jährlich neu diagnostizierten ca. 70000 Patienten mit kolorektalen Karzinomen an ca. 70 Zentren 160 bis 210 „Kolorek-
tal-Chirurgen“, die pro Arbeitstag zwei kolorektale Resektionen durchführen würden, wenn man den Zentrumsgedanken konsequent umsetzen würde und an die damit verbundene Perfektion der chirurgischen Tätigkeit glaubt. Fraglich ist, wie sich die tägliche Routine im Arbeitsalltag auf Dauer auf die Ergebnisse der Spezialisten auswirken würde. Weiterhin ist unklar und zu diskutieren, inwieweit die Spezialisten unser Kliniksystem verändern würden. Unterstehen die Kollegen noch weisungsgebunden ihrem Chefarzt? Wären die Chefärzte bereit, auf diesen Teil ihrer Arbeit zu verzichten?

Könnte die konsequente Zentralisierung der Leistungserbringung nachfolgend zu einer Monopolisierung der Leistungserbringer im Verhältnis gegenüber den Kostenerstattern führen?

Die kritische Sichtung der vorhandenen Literatur zeigt, dass zwar postuliert wird, zertifizierte Krebszentren würden zu einer Qualitätsverbesserung der Behandlung Krebskranker führen bzw. seien dafür unabdingbar. Ein Beweis wurde bisher jedoch nicht erbracht. Die bisher vorliegenden Daten weisen für Darmkrebszentren sogar in die andere Richtung. Insofern stellt sich abschließend die Frage, inwiefern denn das vonseiten der DKG (Organkrebszentren, onkologische Zentren, onkologische Spitzenzentren) und der Fachgesellschaft (Kompetenz-, Referenz-, Exzellenzzentrum) schon etablierte Zentrenranking sinnvoll ist. Festzuhalten bleibt, dass hier finanzielle Ressourcen unseres Gesundheitssystems verschwendet werden, wenn durch Zertifizierung von Darmkrebszentren kein positiver Effekt für die Behandlung der Patienten nachgewiesen werden kann. Dies ist nach $\S 12$ SGBV gesetzeswidrig.

\section{Diskussion}

$\nabla$

Von verschiedener Seite aus fordern Befürworter von Zentren und deren Zertifizierungen, dass Patienten, die an einem kolorektalen Karzinom erkrankt sind, zukünftig möglichst nur noch an zertifizierten Zentren behandelt werden sollten, da lediglich eine Zertifizierung die adäquate Behandlung der Patienten entsprechend den Leitlinien und den geforderten Strukturen sicherstellen könne [1]. Andere Autoren berichten, dass die Datenlage zur chirurgischen Behandlungsqualität in scheinbar überwältigender Weise zeige, dass Faktoren wie die Fallzahl eines Chirurgen, das „High-Volume“-Hospital, eine spezialisierte Ausbildung in kolorektaler Chirurgie und die Überwachung durch Experten eine große Verbesserung für den Patienten darstelle und schließlich in der Bildung spezieller „Colorectal Cancer Units“ münden sollte [11].

Bei kritischer Sichtung der vorliegenden Literatur ergeben sich aber selbst für die komplexen Eingriffe der Ösophagus- und Pankreaschirurgie keine sicher definierbaren „Volume“-Schwellenwerte [21-23]. Da die Effekte eines „High-Volume“-Hospitals oder Chirurgen im Bereich der kolorektalen Chirurgie noch weitaus geringer sind und die Datenlage inkonsistent ist, besteht zum derzeitigen Zeitpunkt keine wissenschaftliche Basis, Mindestmengen im Bereich der kolorektalen Chirurgie festzulegen [4, 6].

Selbstverständlich wird niemand der Forderung widersprechen, dass ein Chirurg, der einen operativen Eingriff durchführt, diesen im Rahmen seiner Weiterbildung zum Chirurgen erlernt haben muss. Ebenso ist jeder Operateur verpflichtet, sich im Rahmen seiner Fortbildung auch neue operative Verfahren, wie damals die TME, anzueignen, um seine Patienten dem aktuellen wissenschaftlichen Stand gemäß bestmöglich zu behandeln. Eine deut- 
liche Verbesserung der Versorgungsqualität von Rektumkarzinompatienten durch Weiterbildung der Chirurgen auch ohne die Bildung von spezialisierten „Colorectal Cancer Units“ und ohne Zertifizierung ist möglich, wie die Daten von Lehander Martling et al. gezeigt haben [15]. Bisher fehlt in der Literatur der Nachweis, dass die arbeitsaufwändige und kostenintensive Zertifizierung von Darmkrebszentren zu einer Verbesserung der Versorgung der Patienten in der Breite geführt hat. Dies wird insbesondere deutlich, wenn die Ergebnisse des Onkozert Benchmark-Berichtes für das Jahr 2010 den Ergebnissen des An-Instituts gegenübergestellt werden [8-10]. Dies ist ein ganz entscheidender Fakt, der auch für die weitere politische Diskussion dieses Themas unbedingt beachtet werden muss, denn die wiederkehrenden Verfahren zur Rezertifizierung verbrauchen nicht unerhebliche finanzielle Ressourcen, deren Einsatz gemäß SGBV nur bei Sinnhaftigkeit gerechtfertigt ist. Es ist durchaus zu diskutieren und zu erforschen, ob durch die Bildung von Krebszentren unter Androhung von Konsequenzen bis hin zum Ausschluss von der Teilnahme an der Patientenversorgung nicht auch nachteilige Effekte auf die chirurgische Ergebnisqualität entstehen können. Beispielhaft ist hier die Auswirkung eines Erfolgsdruckes bei Offenlegung operateursbezogener Behandlungsergebnisse auf Insuffizienzraten und Hartmann'sche Operationen anzuführen. Ebenso ist zu diskutieren, welche Auswirkungen die Zentrenbildung auf die chirurgische Weiterbildung haben werden [21].

Die gesamte Thematik einer Qualitätsverbesserung der Versorgung unserer Patienten durch die Bildung von zertifizierten Zentren ist weitaus komplexer als bisher in der Literatur aufgezeigt. Die begrenzt zur Verfügung stehende Anzahl von Chirurgen mit ihren individuellen Fähigkeiten, die zukünftige flächendeckende Versorgung von Notfallpatienten, die Erhebung justiziabler Datensätze, die Auswirkungen auf unser derzeitiges Kliniksystem und nicht zuletzt die Vergütung von hochspezialisierten Chirurgen ist noch nicht ausreichend diskutiert.

\section{Schlussfolgerung \\ $\nabla$}

Zertifizierungen von Darmkrebszentren sind nur dann sinnvoll, wenn durch sie die Versorgungsqualität der Patienten flächendeckend effektiv gesteigert werden kann. Da anhand der vorliegenden Literatur dieser Vorteil gegenüber nicht zertifizierten Kliniken bisher nicht zu belegen ist, ist der Sinn von Zertifizierungen derzeit zumindest zu hinterfragen. In der weiteren Diskussion über Zentrenbildung und Zertifizierungen sollten die genannten Probleme mit berücksichtigt werden. Die Einhaltung medizinischer Standards und Leitlinien ist der Ärzteschaft aber auch ohne Auditoren und deren Zertifikate möglich.

Interessenkonflikt: Nein.

\section{Literatur}

1 Hohenberger W, Stirkat F, Bruns J et al. Krebsregister und Zentrumsbildung. Der Onkologe 2011; 17: 135-142

2 Coleman MP, Quaresma M, Berrino F et al. Cancer survival in five continents: a worldwide population-based study [CONCORD]. Lancet Oncol 2008; 9: 730-756

3 BGH Urteil (2000) v. 19.04.2000. 3StR 442 / 99, NJW 2000, 2754

4 Roblick UJ, Keller R, Hildebrand $P$ et al. Qualitätsstrukturen und Mindestmengen in der Colon- und Rektumchirurgie. Chirurg 2007; 78: 989-993

5 Greenberg CC, Zinner MJ. Chirurgische Fallzahl - Die amerikanische Perspektive. Chirurg 2007; 78: 1028-1036

6 Weber T, Link KH. Was gibt es Neues bei Mindestmengenvereinbarungen? In: Meßmer K, Jähne J, Neuhaus P, Hrsg. Was gibt es Neues in der Chirurgie? Jahresband; 2011 Heidelberg: Hüthig Jehle Rehm; 2011: $511-520$

7 Grundmann RT. Was gibt es Neues in der präoperativen Risikoabschätzung? In: Meßmer K, Jähne J, Neuhaus P, Hrsg. Was gibt es Neues in der Chirurgie? Jahresband; 2006 Heidelberg: Hüthig Jehle Rehm; 2006; XXIX: $1-3$

8 Deutsche Krebsgesellschaft e.V. [DKG]. Benchmarking 2011 für Darmkrebszentren. Im Internet: www.onkozert.de/aktuelles Stand: 01.06.2011

9 Kube R, Gastinger I, Mroczkowski P et al. Versorgung von Patienten mit Kolonkarzinom. Dtsch Ärztebl Int 2011; 108: 41-46

10 Kube R, Ptok H, Schmidt $U$ et al. Korrelieren die Früh- und Spätergebnisse nach Resektionen des Rektumkarzinoms mit der Anzahl der Eingriffe? Chir Gastroenterol 2008; 24: 293-298

11 Renzulli P, Laffer UT. Learning Curve: The Surgeon as a Prognostic Factor in Colorectal Cancer Surgery. In: Büchler MW, Heald RJ, Ulrich B, Weitz J, Hrsg. Rectal Caner Treatment. Berlin: Springer; 2005: 86-104

12 Smedh K, Olsson L, Johansson $H$ et al. Reduction of postoperative morbidity and mortality in patients with rectal cancer following the introduction of a colorectal unit. Br J Surg 2001; 88: 273-277

13 Machado M, Goldman S, Järhult J et al. Improved results in rectal cancer surgery - an effect of specialization? Colorectal Dis 2000; 2: 264-269

14 Garcia-Granero E, Marti-Obiol R, Gomez-Barbadillo J et al. Impact of surgeon organization and specialization in rectal cancer outcome. Colorectal Dis 2001; 3: 179-184

15 Lehander Martling A, Holm T, Rutqvist L-E et al. Effect of a surgical training programme on outcome of rectal cancer in the County of Stockholm. Lancet 2000; 356: 93-96

16 Kornmann M, Porzsolt F, Henne-Bruns D. Vergleichende Bewertung von Gesundheitsleistungen durch Laien am Beispiel der Therapie des Rektumkarzinoms. Zentralbl Chir 2008; 133: 148-155

17 BVerfG Beschluss (1979) v.25.07.1979. 2 BvR 878/74, NJW 1979, 1925

18 BGH Urteil (2000) v. 15.02.2000 VIZR 48/99, NJW 2000, 1786

19 Ko CY, Chang JT, Chaudhry S et al. Are high-volume surgeons and hospitals the most important predictors oh inhospital outcome for colon cancer resection? Surgery 2002; 132: 268-273

20 Larson DW, Marcello PW, Larach SW et al. Surgeon Volume Does Not Predict Outcomes in the Setting of Technical Credentialing. Ann Surg 2008; 248: 746-750

21 Calasan I, Junger M, Schwendtner $P$ et al. Pankreaschirurgie an einem Krankenhaus der Grund- und Regelversorgung. Zentralbl Chir 2009; 134: 160-165

22 Grundmann RT, Hölscher AH, Bembenek A et al. Diagnostik und Therapie des Magenkarzinoms - Workflow. Zentralbl Chir 2009; 134: 362374

23 Courrech Staal EFW, Coevorden F, Cats A. Outcome of low-volume surgery for esophageal cancer in a high-volume referral center. Ann Surg Oncol 2009; 16: 3219-3226 